\title{
Preferences and Consumption Patterns of Consumer to Develop Processed Pork Products for Export
}

\author{
Sang Hee Seo, Eun Mi Kim*, and Young Boong Kim \\ Korea Food Research Institute, Seongnam 463-746, Korea
}

\begin{abstract}
This study was conducted to survey the perception of domestic consumers about traditional pork dishes, and to utilize the survey result as a basis for the development of processed pork products. A survey was conducted on 218 domestic consumers regarding the following: general questions, propensity and preference to consume pork dishes, pork dishes feasible for export, and the types and problems of processed products feasible for export. The results of the survey showed that the respondents consumed pork dishes 3-4 times a month on average and they appeared to consume the pork dishes most frequently in specialized restaurants. The highest preference was shown in Samgyeopasal gui (grilled pork belly) and Galbi gui (grilled pork rib) among the pork dishes, whereas the lowest preference was shown in Kkubdegi gui (grilled pork skin) and Gookbob (boiled rice and pork served in soup). When the type of processed pork products available according to pork dishes were analyzed, the result showed that Samgyeopasal gui (grilled pork belly) and Nirbiani (slices of roast, seasoned pork in width) could be developed as a frozen product, Geyook Bokeum (stir-fried spicy pork), Bulgogi (Korean-style barbecued pork), and Galbi jjim (braised short ribs) could be developed as a retort product, and that Bossam (boiled pork eaten with a salty sauce and wrapped in greens), Pyeonyuk (slices of boiled pork), Jokbal (pork trotters), and Sundae (kneading together seasoned mixture of vegetables and glass noodles stuffing it into casings made of hog intestines, which are then tied off at the ends and boiled) could be developed as a vacuum-packed product. When the survey concerning the problems expected in the development of processed products using pork dishes was conducted, the result showed that there could be a texture change issue for Galbi gui, Samgyeopasal gui and Nirbiani; a preservation issue for Bossam, Pyeonyuk; a safety issue for Bulgogi and Geyook Bokeum; a taste and flavor change issue for Geyook Bokeum and Sundae; and a packaging issue for Gookbob.
\end{abstract}

Key words: pork dishes, preference, consumption patterns, processed pork product

\section{Introduction}

Import liberalization was executed due to FTA between Korea and Chile in 2004, and FTA between Korea and USA was concluded in 2007. In addition, simultaneous FTA negotiations and pre-meetings for FTA negotiations with advanced countries for livestock industry are currently in progress. Accordingly, not only domestic livestock industry but also distribution and processing industries are expected to be damaged from increased import. In addition, domestic consumers are also concerned about the safety and hygienic condition of foreign livestocks. For the past several years, Korean government has invested a lot of funds and established policies on the improvement of production and distribution channel for reinforc-

*Corresponding author: Eun-Mi Kim, Korea Food Research Institute, Seongnam 463-746, Korea. Tel: 82-31-780-9287, Fax: 82-31-780-9076, E-mail: kem@kfri.re.kr ing the competitiveness of domestic livestock industry to measure import liberalization. However, the policies have mainly focused on primary industries, which resulted in relatively less focusing on the investment in livestock processing industry (Lim, 2009).

According to the present condition of exportation and importation of processed meat products published by the Korea meat industries association, the import amount in 2008 was 12,997 ton, increasing by $24.5 \%$, and the export amount was 144.5 ton, increasing by $10.6 \%$ compared to the previous year. However, since the outbreak of footand-mouth disease in 2000, the stagnant consumption of processed pork products has been continued due to the following reasons such as the accumulation of non-preferred meat parts due to the halt of pork export to Japan, unstable demand and supply due to Samgyeopasal based food culture, negative perception of consumers regarding processed meat products containing sodium nitrite, and the restriction of consumption expansion due to different 
policies from those of developed countries (Lim, 2009).

Accordingly, this study was conducted to investigate the perception, preference and consumption pattern of domestic consumers about pork dishes (Bossam : boiled pork eaten with a salty sauce and wrapped in greens, Pyeonyuk : slices of boiled pork, Jokbal : pork trotters, Bulgogi : Korean-style barbecued pork, Galbi jjim : braised short ribs, Galbi gui : grilled pork rib, Samgyeopasal gui : grilled pork belly, Nirbiani : slices of roast, seasoned pork in width, Kkubdegi gui : grilled pork skin, Gobchang gui : grilled small intestine, Gookbob : boiled rice and pork served in soup, Sundae : kneading together seasoned mixture of vegetables and glass noodles stuffing it into casings made of pig intestines, which are then tied off at the ends and boiled, Geyook Bokeum : stir-fried spicy pork) and to utilize the survey result as a basis for the development of processed pork products for export using pork consumed the most in the world.

\section{Methods}

\section{Survey subjects and duration}

The questionnaire consisted of questions designed for this study by referring to previous references. A preliminary survey was conducted on researchers working in the institute and a primary study was conducted after modifying and supplementing the preliminary survey. The survey was conducted on 218 residents in Kyunggi province from April 2010 to June 2010.

\section{Survey methods and contents}

A total of 227 questionnaires were distributed, of which 218 questionnaires was returned (return rate 96.0\%). After surveyors explained survey backgrounds to respondents, the respondents completed the questionnaire in a self-reporting manner. The questionnaire consisted of questions asking general information, the preference and propensity of consumers, export feasibility, the types and problems of products feasible for export. The preference for pork dishes and the feasibility for export were assessed using a 5-point scale. The types and problems of products feasible for export were surveyed by answering to the question (multiple answering). The preference was assessed as follows: 1 : very dislike, 3 : so so, and 5: very like. The export feasibility was assessed as follows: 1 : very low, 3 : so so, and 5: very high.

\section{Data analysis}

Descriptive analysis, ANOVA, independent samples t- test, and correspondence analysis were conducted on data collected using SPSS 12.0. The results were denoted as the mean $\pm \mathrm{SD}$, and Duncan's multiple range test was conducted for Post-Hoc test.

\section{Results and Discussion}

\section{General information on the subjects}

The respondents, who participated in the survey, consisted of 79 men (36.2\%) and 139 women (63.8\%). 88 respondents (40.4\%) belonged to age of 40-49 years and 65 respondents $(29.8 \%)$ belonged to age of 29 years or less in order. 136 respondents $(62.4 \%)$ were in married status and 80 respondents $(36.7 \%)$ were in unmarried status. 69 respondents (31.7\%) were housewives, 65 respondents $(29.8 \%)$ were office workers, and 56 respondents $(25.7 \%)$ were professionals in order (Table 1).

\section{Consumption propensity of the subjects regarding pork dishes}

The result of the survey on the consumption propensity of the subjects regarding pork dishes was presented in Table 2. For consumption frequency, 87 respondents (39.9\%) responded that they consumed pork dishes 3-4 times per month on average. For consuming place, the subjects responded in the following order : specialized restaurant $(37.1 \%)>$ home $(35.7 \%)>$ general restaurant $(21.4 \%)$. The average expense of less than 10,000 won per person accounted for the highest portion of the subjects, and the most preferred cooking method was identi-

Table 1. General characteristics of respondents

\begin{tabular}{|c|c|c|c|}
\hline \multicolumn{2}{|c|}{ Characteristics } & Frequency & $\%$ \\
\hline \multirow{2}{*}{ Gender } & Male & 79 & 36.2 \\
\hline & Female & 139 & 63.8 \\
\hline \multirow{4}{*}{ Age } & $<30$ & 65 & 29.8 \\
\hline & $30-40$ & 55 & 25.2 \\
\hline & $40-50$ & 88 & 40.4 \\
\hline & $\geq 50$ & 10 & 4.6 \\
\hline \multirow{3}{*}{ Marriage status } & Single & 80 & 36.7 \\
\hline & Married & 136 & 62.4 \\
\hline & No response & 2 & 0.9 \\
\hline \multirow{8}{*}{ Occupation } & Student & 6 & 2.8 \\
\hline & Office worker & 65 & 29.8 \\
\hline & Housewife & 69 & 31.7 \\
\hline & Self-employed & 11 & 5.0 \\
\hline & School person & 3 & 1.4 \\
\hline & Profession & 56 & 25.7 \\
\hline & Others & 5 & 2.3 \\
\hline & No response & 3 & 1.4 \\
\hline
\end{tabular}


Table 2. Consumption patterns of pork dishes

\begin{tabular}{|c|c|c|c|}
\hline \multicolumn{2}{|c|}{ Characteristics } & \multirow{2}{*}{$\begin{array}{c}\text { Frequency } \\
40\end{array}$} & \multirow{2}{*}{$\begin{array}{c}\% \\
18.3\end{array}$} \\
\hline & $1-2$ & & \\
\hline & $3-4$ & 87 & 39.9 \\
\hline \multirow{4}{*}{$\begin{array}{l}\text { Intake frequency } \\
\text { (per month) }\end{array}$} & $5-6$ & 52 & 23.9 \\
\hline & $7-8$ & 22 & 10.1 \\
\hline & Over 9 & 14 & 6.4 \\
\hline & No response & 3 & 1.4 \\
\hline \multirow{5}{*}{$\begin{array}{c}\text { Eating place } \\
\text { (multiple } \\
\text { answers) }\end{array}$} & Family restaurant & 8 & 2.3 \\
\hline & General restaurant & 75 & 21.4 \\
\hline & Home & 125 & 35.7 \\
\hline & Specialized restaurant & 130 & 37.1 \\
\hline & School (Office) cafeteria & 12 & 3.4 \\
\hline \multirow{5}{*}{$\begin{array}{c}\text { Actual expense } \\
\text { (won/person) }\end{array}$} & $<10000$ & 145 & 66.5 \\
\hline & $1-20000$ & 57 & 26.1 \\
\hline & $2-30000$ & 13 & 6.0 \\
\hline & $\geq 30000$ & 1 & 0.5 \\
\hline & No response & 2 & 0.9 \\
\hline \multirow{6}{*}{$\begin{array}{c}\text { Preferred } \\
\text { cooking method }\end{array}$} & Roast & 152 & 69.7 \\
\hline & Stir fry & 47 & 21.6 \\
\hline & Deep fry & 1 & 0.5 \\
\hline & Steam & 11 & 5.0 \\
\hline & Stew & 4 & 1.8 \\
\hline & No response & 3 & 1.4 \\
\hline \multirow{8}{*}{$\begin{array}{l}\text { Important fac- } \\
\text { tors to consider } \\
\quad \text { (score) }\end{array}$} & Items & $\mathrm{M} \pm \mathrm{SD}$ & Rank \\
\hline & Taste & $6.09 \pm 1.18$ & 1 \\
\hline & Health & $3.68 \pm 1.37$ & 5 \\
\hline & Moderate amount & $3.17 \pm 1.27$ & 6 \\
\hline & Price & $4.03 \pm 1.42$ & 4 \\
\hline & Freshness & $5.45 \pm 1.18$ & 2 \\
\hline & Provenance & $4.56 \pm 1.82$ & 3 \\
\hline & Others (Aroma, safety) & $0.35 \pm 0.99$ & 7 \\
\hline
\end{tabular}

fied to be roast (gui). This result was similar to that of a study conducted by Kim et al. (2010) reporting that the most preferred cooking method was identified to be roast (gui) in the cases of meat dishes. Upon the selection of pork dishes, factors were considered to be important in the following order : taste $>$ freshness $>$ provenance (place of origin) $>$ price $>$ health $>$ amount. This result was also similar to that of the study conducted by Kim et al. (2010) reporting that taste was the number one priority upon the selection of meat dishes.

\section{Preference for pork dishes}

When preference for pork dishes was surveyed, higher preference was shown in Samgyeopasal gui (4.18) and Galbi gui (4.13). This result was similar to that of a study conducted by Kwak and Ko (2006) reporting that Pork galbi (46.0\%) and Samgyeopasal gui (40.5\%) were the most frequently selected upon going out to eat with fam- ily, and those of studies conducted by Kim and Kim (2009), and Kim et al. (2010). This result is likely to be attributable to the fact that Samgyeopasal, Moksal (boston butt), and Galbi are mainly consumed for roast (gui) in the domestic consumption pattern of pork. On the contrary, the preference for Kkubdegi gui (2.44) and Gookbob (2.60) were shown to be low (Table3). This result was consistent with that of a study conducted by Choi (2009) reporting that the highest preference was shown in Samgyeopasal gui and the lowest preference was shown in gookbob.

When the preference for pork dishes was surveyed according to gender, a significant difference was found in Kkubdegi gui $(p<0.05)$, Gobchang gui $(p<0.05)$, and Gookbob $(p<0.001)$. However, high preference for Samgyeopasal gui and Galbi gui was shown regardless of gender.

When the preference for pork dishes was surveyed according to age, below 30 age group had a high preference in Jokbal, Kkubdegi gui, Gobchang gui $(p<0.01)$, Bulgogi, Galbi jjim, and Nirbiani $(p<0.05)$ compared with other age groups.

\section{Pork dishes feasible for export}

When pork dishes feasible for export was surveyed, the feasibility was shown to be high in Galbi gui (4.07), Galbi jjim (4.06), and Nirbiani (4.00). Meanwhile, the feasibility was shown to be low in Gookbob (2.19), Kkubdegi gui (2.32), Gobchang gui (2.35) (Table 4).

When the feasibility for export was surveyed according to gender, the feasibility was shown to be high in Galbi jjim (4.00), Galbi gui (3.97), and Bulgogi (3.85) in order for men and in Nirbiani (4.13), Galbi jjim (4.13), and Galbi gui (4.09) in order for women. A significant difference was found in Nirbiani $(p<0.01)$ and Kkubdegi gui $(p<0.05)$ between men and women.

When the feasibility for export was surveyed according to age, a significant difference was found in Bulgogi, Nirbiani, and Geyook bokeum $(p<0.01)$. For age less than 30 years, the highest feasibility was shown in Nirbiani (4.37). For age of 30-40, 40-50 years, the highest feasibility was shown in Galbi gui (4.20 and 4.00, respectively). For age of 50 years or higher, the highest feasibility was shown in Galbi jjim (4.30).

\section{Types and problems of the processed pork dishes feasible for export}

The result of analyzing the types of the processed pork dishes feasible for export was presented in Fig. 1. Correspondence analysis shows correlation between factors on 
Table 3. Comsumer's preference of pork dishes

$(\mathrm{M} \pm \mathrm{SD})$

\begin{tabular}{|c|c|c|c|c|c|c|c|c|c|c|c|c|}
\hline Items $^{1)}$ & $\begin{array}{c}\text { Bossam, } \\
\text { Pyeonyuk }\end{array}$ & Jokbal & Bulgogi & Galbi jjim & Galbigui & $\begin{array}{l}\text { Samgyeop- } \\
\text { sal gui }\end{array}$ & Nirbiani & $\begin{array}{c}\text { Kkubdegi } \\
\text { gui }\end{array}$ & $\begin{array}{c}\text { Gobchang } \\
\text { gui }\end{array}$ & Gookbob & Sundae & $\begin{array}{l}\text { Geyook } \\
\text { Bokeum }\end{array}$ \\
\hline $\begin{array}{l}\text { Prefer- } \\
\text { ence (n) }\end{array}$ & $\begin{array}{c}3.87 \pm \\
0.87 \\
(217) \\
\end{array}$ & $\begin{array}{c}3.59 \pm \\
0.99 \\
(217) \\
\end{array}$ & $\begin{array}{c}3.75 \pm \\
0.87 \\
(217) \\
\end{array}$ & $\begin{array}{c}3.86 \pm \\
0.84 \\
(216)\end{array}$ & $\begin{array}{c}4.13 \pm \\
0.82 \\
(216) \\
\end{array}$ & $\begin{array}{c}4.18 \pm \\
0.90 \\
(217) \\
\end{array}$ & $\begin{array}{c}3.36 \pm \\
1.02 \\
(212)\end{array}$ & $\begin{array}{c}2.44 \pm \\
1.16 \\
(214) \\
\end{array}$ & $\begin{array}{c}2.91 \pm \\
1.33 \\
(214) \\
\end{array}$ & $\begin{array}{c}2.60 \pm \\
1.22 \\
(214)\end{array}$ & $\begin{array}{c}3.65 \pm \\
0.91 \\
(216)\end{array}$ & $\begin{array}{c}3.84 \pm \\
0.90 \\
(217)\end{array}$ \\
\hline \multicolumn{13}{|l|}{ Gender } \\
\hline Male (n) & $\begin{array}{c}3.96 \pm \\
0.83 \\
(78)\end{array}$ & $\begin{array}{c}3.72 \pm \\
0.95 \\
(78)\end{array}$ & $\begin{array}{c}3.77 \pm \\
0.91 \\
(78)\end{array}$ & $\begin{array}{c}3.87 \pm \\
0.91 \\
(77)\end{array}$ & $\begin{array}{c}4.04 \pm \\
0.87 \\
(77)\end{array}$ & $\begin{array}{c}4.23 \pm \\
0.82 \\
(78)\end{array}$ & $\begin{array}{c}3.28 \pm \\
0.94 \\
(75)\end{array}$ & $\begin{array}{c}2.69 \pm \\
1.07 \\
(77)\end{array}$ & $\begin{array}{c}3.22 \pm \\
1.21 \\
(76)\end{array}$ & $\begin{array}{c}3.01 \pm \\
1.08 \\
(76)\end{array}$ & $\begin{array}{c}3.57 \pm \\
0.95 \\
(77)\end{array}$ & $\begin{array}{c}3.88 \pm \\
0.87 \\
(78)\end{array}$ \\
\hline $\begin{array}{c}\text { Female } \\
\text { (n) }\end{array}$ & $\begin{array}{c}3.82 \pm \\
0.88 \\
(139)\end{array}$ & $\begin{array}{c}3.51 \pm \\
1.01 \\
(139)\end{array}$ & $\begin{array}{c}3.73 \pm \\
0.85 \\
(139)\end{array}$ & $\begin{array}{c}3.85 \pm \\
0.81 \\
(139)\end{array}$ & $\begin{array}{c}4.19 \pm \\
0.80 \\
(139)\end{array}$ & $\begin{array}{c}4.15 \pm \\
0.94 \\
(139)\end{array}$ & $\begin{array}{c}3.41 \pm \\
1.03 \\
(137)\end{array}$ & $\begin{array}{c}2.31 \pm \\
1.20 \\
(137)\end{array}$ & $\begin{array}{c}2.74 \pm \\
1.36 \\
(138)\end{array}$ & $\begin{array}{c}2.38 \pm \\
1.23 \\
(138)\end{array}$ & $\begin{array}{c}3.70 \pm \\
0.88 \\
(139)\end{array}$ & $\begin{array}{c}3.81 \pm \\
0.92 \\
(139)\end{array}$ \\
\hline T-value & 1.15 & 1.48 & 0.29 & 0.18 & -1.27 & 0.63 & -0.88 & $2.33^{*}$ & $2.59^{*}$ & $3.93^{* * *}$ & 0.98 & 0.56 \\
\hline \multicolumn{13}{|l|}{ Ages } \\
\hline $\begin{array}{l}<30 \\
(\mathrm{n})\end{array}$ & $\begin{array}{c}3.97 \pm \\
0.85 \\
(65) \\
\end{array}$ & $\begin{array}{c}3.82 \pm \\
1.01 \\
(65) \\
\end{array}$ & $\begin{array}{c}4.00 \pm \\
0.75 \\
(65) \\
\end{array}$ & $\begin{array}{c}4.09 \pm \\
0.74 \\
(65) \\
\end{array}$ & $\begin{array}{c}4.31 \pm \\
0.71 \\
(65) \\
\end{array}$ & $\begin{array}{c}4.22 \pm \\
0.98^{\mathrm{ab}} \\
(65) \\
\end{array}$ & $\begin{array}{c}3.69 \pm \\
1.02^{\mathrm{b}} \\
(64) \\
\end{array}$ & $\begin{array}{c}2.78 \pm \\
1.20 \\
(64) \\
\end{array}$ & $\begin{array}{c}3.38 \pm \\
1.40^{\mathrm{b}} \\
(64)\end{array}$ & $\begin{array}{c}2.88 \pm \\
1.24 \\
(65) \\
\end{array}$ & $\begin{array}{c}3.80 \pm \\
0.89 \\
(65) \\
\end{array}$ & $\begin{array}{c}3.86 \pm \\
0.98 \\
(65) \\
\end{array}$ \\
\hline $\begin{array}{c}30-40 \\
(\mathrm{n}=54)\end{array}$ & $\begin{array}{c}3.98 \pm \\
0.88\end{array}$ & $\begin{array}{c}3.80 \pm \\
0.79\end{array}$ & $\begin{array}{c}3.76 \pm \\
0.87\end{array}$ & $\begin{array}{c}3.81 \pm \\
0.91\end{array}$ & $\begin{array}{c}4.11 \pm \\
0.86\end{array}$ & $\begin{array}{l}4.33 \pm \\
0.78^{\mathrm{b}}\end{array}$ & $\begin{array}{l}3.22 \pm \\
1.11^{\mathrm{ab}}\end{array}$ & $\begin{array}{c}2.57 \pm \\
1.16\end{array}$ & $\begin{array}{l}2.96 \pm \\
1.40^{\mathrm{ab}}\end{array}$ & $\begin{array}{c}2.65 \pm \\
1.18\end{array}$ & $\begin{array}{c}3.80 \pm \\
0.88\end{array}$ & $\begin{array}{c}3.96 \pm \\
0.87\end{array}$ \\
\hline $\begin{array}{c}40-50 \\
(\mathrm{n})\end{array}$ & $\begin{array}{c}3.72 \pm \\
0.88 \\
(88)\end{array}$ & $\begin{array}{c}3.31 \pm \\
1.02 \\
(88)\end{array}$ & $\begin{array}{c}3.53 \pm \\
0.92 \\
(88)\end{array}$ & $\begin{array}{c}3.71 \pm \\
0.84 \\
(87)\end{array}$ & $\begin{array}{c}4.02 \pm \\
0.88 \\
(87)\end{array}$ & $\begin{array}{c}4.10 \pm \\
0.91^{\mathrm{ab}} \\
(88)\end{array}$ & $\begin{array}{c}3.26 \pm \\
0.94^{\mathrm{ab}} \\
(86)\end{array}$ & $\begin{array}{c}2.14 \pm \\
1.08 \\
(86)\end{array}$ & $\begin{array}{c}2.57 \pm \\
1.20^{\mathrm{a}} \\
(86)\end{array}$ & $\begin{array}{c}2.41 \pm \\
1.22 \\
(86)\end{array}$ & $\begin{array}{c}3.48 \pm \\
0.92 \\
(88)\end{array}$ & $\begin{array}{c}3.78 \pm \\
0.85 \\
(88)\end{array}$ \\
\hline $\begin{array}{l}\geq 50 \\
\text { (n) }\end{array}$ & $\begin{array}{c}4.00 \pm \\
0.67 \\
(10)\end{array}$ & $\begin{array}{c}3.40 \pm \\
0.84 \\
(10)\end{array}$ & $\begin{array}{c}3.90 \pm \\
0.74 \\
(10)\end{array}$ & $\begin{array}{c}3.80 \pm \\
0.79 \\
(10)\end{array}$ & $\begin{array}{c}4.10 \pm \\
0.74 \\
(10)\end{array}$ & $\begin{array}{c}3.80 \pm \\
0.79^{\mathrm{a}} \\
(10)\end{array}$ & $\begin{array}{c}2.88 \pm \\
0.64^{\mathrm{a}} \\
(8)\end{array}$ & $\begin{array}{c}2.20 \pm \\
1.14 \\
(10)\end{array}$ & $\begin{array}{c}2.60 \pm \\
0.84^{\mathrm{a}} \\
(10)\end{array}$ & $\begin{array}{c}2.22 \pm \\
0.83 \\
(9)\end{array}$ & $\begin{array}{c}3.44 \pm \\
0.88 \\
(9)\end{array}$ & $\begin{array}{c}3.50 \pm \\
0.97 \\
(10)\end{array}$ \\
\hline F-value & 1.596 & $4.627 * *$ & $3.852^{*}$ & $2.663^{*}$ & 1.529 & 1.384 & $3.562 *$ & $4.312^{* *}$ & $4.975^{* *}$ & 2.198 & 2.319 & 0.934 \\
\hline
\end{tabular}

5 scale (1, dislike extremely; 5 , like extremely)

${ }^{*} p<0.05, * * p<0.01, * * * p<0.001$

${ }^{\mathrm{ab}}$ Duncan's multiple range test

${ }^{1)}$ Bossam, boiled pork eaten with a salty sauce and wrapped in greens; Pyeonyuk, slices of boiled pork; Jokbal, pork trotters; Bulgogi, Korean-style barbecued pork; Galbi jjim, braised short ribs; Galbi gui, grilled pork rib; Samgyeopasal gui, grilled pork belly; Nirbiani, slices of roast, seasoned pork in width; Kkubdegi gui, grilled pork skin; Gobchang gui, grilled small intestine; Gookbob, boiled rice and pork served in soup; Sundae, kneading together seasoned mixture of vegetables and glass noodles stuffing it into casings made of pig intestines which are then tied off at the ends and boiled; Geyook Bokeum, stir-fried spicy pork

a 2-dimensional map. The correlation is high if between point's distance is close.

For the types of the processed pork dishes feasible for export, the result showed that Samgyeopasal gui and Nirbiani could be developed as a frozen product; Geyook bokeum, Bulgogi, and Galbi jjim as a retort product; Gookbob could as a can product; and Bossam, Pyeonyuk, Sundae, and Jokbal as a vacuum-packed product.

For the problems of the processed pork dishes feasible for export, the result showed that texture change could be problematic in Galbi gui, Samgyeopasal gui, and Nirbiani; preservation could be problematic in Bossam and Pyeonyuk; safety (food additives) could be problematic in Bulgogi and Geyook bokeum; changes in taste and flavor could be problematic in Galbi jjim and Sundae; and packing could be problematic in Gookbob (Fig. 2). The afore- mentioned results were similar to those of a study conducted by Yoon (2003) reporting that reasons of not trusting processed meat products were shown to be containing of additives and hazardous chemicals (44.1\%), amount and quality (28.7\%), and preservation condition (15.2\%) in order. Thus, the aforementioned results showed that hygiene and health such as additives and hazardous chemicals were major concerns.

\section{Conclusion}

This study was conducted to survey the perception of domestic consumers about traditional pork dishes, and to utilize the survey result as a basis for the development of processed pork products using traditional pork dishes. Among the pork dishes, higher preference was shown in 
Table 4. Feasibility score of processed pork products for export

$(\mathrm{M} \pm \mathrm{SD})$

\begin{tabular}{|c|c|c|c|c|c|c|c|c|c|c|c|c|}
\hline Items $^{1)}$ & $\begin{array}{l}\text { Bossam, } \\
\text { Pyeonyuk }\end{array}$ & okbal & ulgogi & Galbi jjim & Galbigui & $\begin{array}{c}\text { Samgyeo- } \\
\text { psal gui }\end{array}$ & Nirbiani & $\begin{array}{c}\text { Kkubdegi } \\
\text { gui }\end{array}$ & $\begin{array}{c}\text { Gob- } \\
\text { chang gui }\end{array}$ & Gookbob & Sundae & $\begin{array}{l}\text { Geyook } \\
\text { Bokeum }\end{array}$ \\
\hline $\begin{array}{c}\text { Feasibili- } \\
\text { ty }^{2)}(n)\end{array}$ & $\begin{array}{c}3.67 \pm \\
0.89 \\
(217)\end{array}$ & $\begin{array}{c}3.67 \pm \\
1.00 \\
(216)\end{array}$ & $\begin{array}{c}3.95 \pm \\
0.90 \\
(215)\end{array}$ & $\begin{array}{c}4.06 \pm \\
0.89 \\
(216)\end{array}$ & $\begin{array}{c}4.07 \pm \\
0.94 \\
(214)\end{array}$ & $\begin{array}{c}3.42 \pm \\
1.13 \\
(216)\end{array}$ & $\begin{array}{c}4.00 \pm \\
0.99 \\
(216)\end{array}$ & $\begin{array}{c}2.32 \pm \\
1.01 \\
(213)\end{array}$ & $\begin{array}{c}2.35 \pm \\
0.96 \\
(212)\end{array}$ & $\begin{array}{c}2.19 \pm \\
0.93 \\
(213)\end{array}$ & $\begin{array}{c}3.29 \pm \\
1.12 \\
(215)\end{array}$ & $\begin{array}{c}3.61 \pm \\
0.86 \\
(217)\end{array}$ \\
\hline \multicolumn{13}{|l|}{ Gender } \\
\hline Mal & $\begin{array}{c}3.66 \pm \\
1.04 \\
(79)\end{array}$ & $\begin{array}{c}3.72 \pm \\
1.00 \\
(79)\end{array}$ & $\begin{array}{c}3.85 \pm \\
0.96 \\
(78)\end{array}$ & $\begin{array}{c}4.00 \pm \\
0.95 \\
(78)\end{array}$ & $\begin{array}{c}3.97 \pm \\
0.99 \\
(78)\end{array}$ & $\begin{array}{c}3.49 \pm \\
1.12 \\
(79)\end{array}$ & $\begin{array}{c}3.76 \pm \\
1.08 \\
(78)\end{array}$ & $\begin{array}{c}2.51 \pm \\
1.11 \\
(77)\end{array}$ & $\begin{array}{c}2.48 \pm \\
0.97 \\
(77)\end{array}$ & $\begin{array}{c}2.31 \pm \\
1.03 \\
(77)\end{array}$ & $\begin{array}{c}3.24 \pm \\
3.32 \\
(78)\end{array}$ & $\begin{array}{c}3.62 \pm \\
0.95 \\
(79)\end{array}$ \\
\hline $\begin{array}{c}\text { Female } \\
\text { (n) }\end{array}$ & $\begin{array}{c}3.64 \pm \\
0.85 \\
(138)\end{array}$ & $\begin{array}{c}3.58 \pm \\
1.09 \\
(137)\end{array}$ & $\begin{array}{c}4.01 \pm \\
0.87 \\
(138)\end{array}$ & $\begin{array}{c}4.09 \pm \\
0.85 \\
(138)\end{array}$ & $\begin{array}{c}4.13 \pm \\
0.90 \\
(136)\end{array}$ & $\begin{array}{c}3.37 \pm \\
1.14 \\
(137)\end{array}$ & $\begin{array}{c}4.13 \pm \\
0.90 \\
(138)\end{array}$ & $\begin{array}{c}2.21 \pm \\
0.95 \\
(136)\end{array}$ & $\begin{array}{c}2.28 \pm \\
0.96 \\
(135)\end{array}$ & $\begin{array}{c}2.13 \pm \\
0.86 \\
(136)\end{array}$ & $\begin{array}{c}3.32 \pm \\
1.10 \\
(137)\end{array}$ & $\begin{array}{c}3.61 \pm \\
0.81 \\
(138)\end{array}$ \\
\hline t-value & -0.12 & 0.61 & -1.32 & -0.75 & -1.19 & 0.76 & $-2.72^{* *}$ & $2.04^{*}$ & 1.45 & 1.41 & -0.49 & 0.10 \\
\hline \multicolumn{13}{|l|}{ Ages } \\
\hline $\begin{array}{l}<30 \\
\text { (n) }\end{array}$ & $\begin{array}{c}3.66 \pm \\
0.91 \\
(65)\end{array}$ & $\begin{array}{c}3.62 \pm \\
1.09 \\
(65)\end{array}$ & $\begin{array}{c}4.30 \pm \\
0.85^{\mathrm{b}} \\
(64)\end{array}$ & $\begin{array}{c}4.23 \pm \\
0.77 \\
(64)\end{array}$ & $\begin{array}{c}4.08 \pm \\
0.94 \\
(63)\end{array}$ & $\begin{array}{c}3.45 \pm \\
1.19 \\
(64)\end{array}$ & $\begin{array}{c}4.37 \pm \\
0.74^{\mathrm{b}} \\
(65)\end{array}$ & $\begin{array}{c}2.14 \pm \\
0.91 \\
(64)\end{array}$ & $\begin{array}{c}2.28 \pm \\
1.00 \\
(64)\end{array}$ & $\begin{array}{c}2.19 \pm \\
0.81 \\
(64)\end{array}$ & $\begin{array}{c}3.15 \pm \\
1.20 \\
(65)\end{array}$ & $\begin{array}{c}3.89 \pm \\
0.81^{\mathrm{b}} \\
(65)\end{array}$ \\
\hline $\begin{array}{c}30-40 \\
\text { (n) }\end{array}$ & $\begin{array}{c}3.80 \pm \\
0.83 \\
(55)\end{array}$ & $\begin{array}{c}3.74 \pm \\
1.05 \\
(54)\end{array}$ & $\begin{array}{c}3.98 \pm \\
0.90^{\mathrm{ab}} \\
(54)\end{array}$ & $\begin{array}{c}4.07 \pm \\
1.03 \\
(55)\end{array}$ & $\begin{array}{c}4.20 \pm \\
0.90 \\
(54)\end{array}$ & $\begin{array}{c}3.46 \pm \\
1.08 \\
(54)\end{array}$ & $\begin{array}{c}3.81 \pm \\
1.15^{\mathrm{a}} \\
(54)\end{array}$ & $\begin{array}{c}2.24 \pm \\
0.78 \\
(54)\end{array}$ & $\begin{array}{c}2.34 \pm \\
1.00 \\
(53)\end{array}$ & $\begin{array}{c}2.13 \pm \\
0.91 \\
(54)\end{array}$ & $\begin{array}{c}3.26 \pm \\
1.07 \\
(54)\end{array}$ & $\begin{array}{c}3.69 \pm \\
0.84^{\mathrm{ab}} \\
(54)\end{array}$ \\
\hline $\begin{array}{l}40-50 \\
\text { (n) }\end{array}$ & $\begin{array}{c}3.59 \pm \\
0.94 \\
(87)\end{array}$ & $\begin{array}{c}3.64 \pm \\
0.93 \\
(87)\end{array}$ & $\begin{array}{c}3.71 \pm \\
0.86^{\mathrm{a}} \\
(87)\end{array}$ & $\begin{array}{c}3.90 \pm \\
0.88 \\
(87)\end{array}$ & $\begin{array}{c}4.00 \pm \\
0.94 \\
(87)\end{array}$ & $\begin{array}{c}3.35 \pm \\
1.12 \\
(88)\end{array}$ & $\begin{array}{c}3.90 \pm \\
0.94^{\mathrm{ab}} \\
(87)\end{array}$ & $\begin{array}{c}2.48 \pm \\
1.16 \\
(85)\end{array}$ & $\begin{array}{c}2.45 \pm \\
0.92 \\
(85)\end{array}$ & $\begin{array}{c}2.21 \pm \\
0.99 \\
(85)\end{array}$ & $\begin{array}{c}3.37 \pm \\
1.07 \\
(86)\end{array}$ & $\begin{array}{c}3.40 \pm \\
0.84^{\mathrm{a}} \\
(88)\end{array}$ \\
\hline $\begin{array}{c}\geq 50 \\
(\mathrm{n}=10)\end{array}$ & $\begin{array}{c}3.70 \pm \\
0.68\end{array}$ & $\begin{array}{c}3.80 \pm \\
0.92\end{array}$ & $\begin{array}{l}3.70 \pm \\
0.95^{\mathrm{a}}\end{array}$ & $\begin{array}{c}4.30 \pm \\
0.68\end{array}$ & $\begin{array}{c}4.00 \pm \\
1.16\end{array}$ & $\begin{array}{c}3.50 \pm \\
1.27\end{array}$ & $\begin{array}{l}3.40 \pm \\
1.17^{\mathrm{a}}\end{array}$ & $\begin{array}{c}2.50 \pm \\
1.35\end{array}$ & $\begin{array}{c}2.10 \pm \\
1.00\end{array}$ & $\begin{array}{c}2.40 \pm \\
1.27\end{array}$ & $\begin{array}{c}3.70 \pm \\
1.34\end{array}$ & $\begin{array}{l}3.30 \pm \\
0.95^{\mathrm{a}}\end{array}$ \\
\hline F-value & 0.654 & 0.227 & $5.827 * *$ & 2.075 & 0.544 & 0.164 & $5.558 * *$ & 1.621 & 0.616 & 0.259 & 0.926 & $4.958^{* *}$ \\
\hline
\end{tabular}

${ }^{1)}$ Refer to Table 3

2) 5 scale (1, very low; 5 , very high)

$* p<0.05 * * p<0.01$

${ }^{\mathrm{ab}}$ Duncan's multiple range test

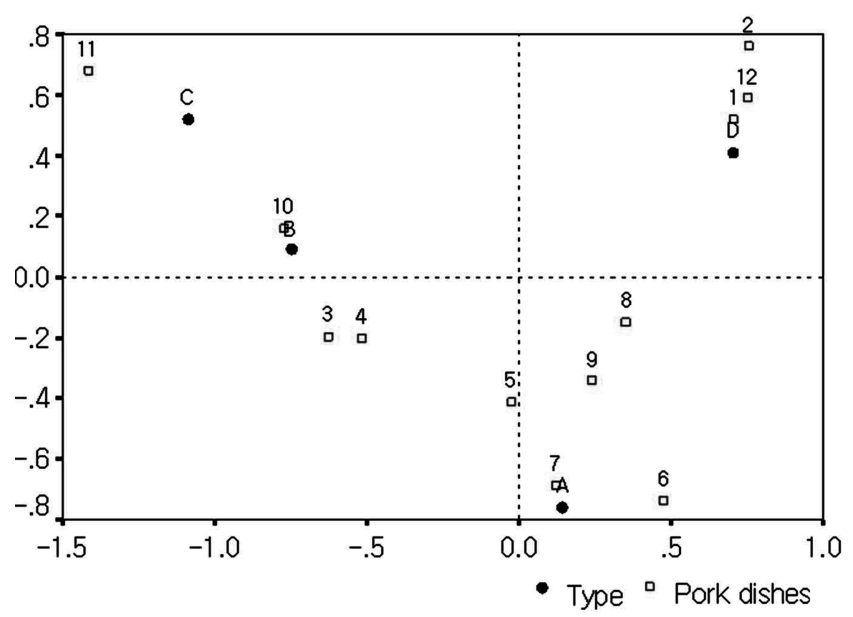

Fig. 1. Correspondence-plot of processing type by pork dishes. 1, Bossam, Pyeonyuk; 2, Jokbal; 3, Bulgogi; 4, Galbi jjim; 5, Galbi gui; 6, Samgyeopsal gui; 7, Nirbiani; 8, Kkubdegi gui; 9, Gobchang gui; 10, Geyook Bokeum; 11, Gookbob; 12, Sundae. A, Frozen product; B, Retort; C, Can; D, Vacuum wrapping

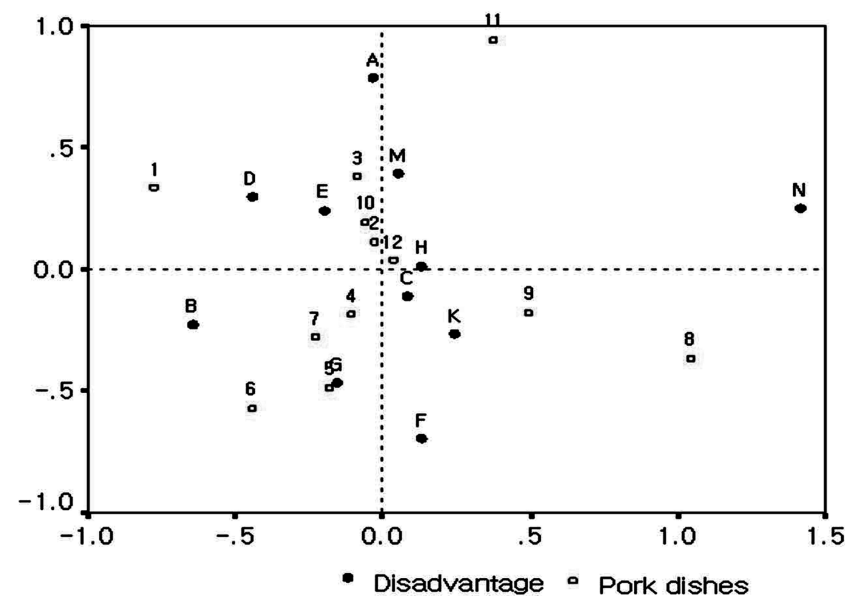

Fig. 2. Correspondence-plot of disadvantage by pork dishes. 1, Bossam, Pyeonyuk; 2, Jokbal; 3, Bulgogi; 4, Galbi jjim; 5, Galbi gui; 6, Samgyeopsal gui; 7, Nirbiani; 8, Kkubdegi gui; 9, Gobchang gui; 10, Geyook Bokeum; 11, Gookbob; 12, Sundae. A, Packaging; B, Price; C, Taste; D, Conservativeness (storability); E, Safety (food additives); F, Cooking convenience; G, Texture changes; H, Aroma changes; K, Size of product; M, Destroying nutrients; N, Type of contents 
Samgyeopasal gui and Galbi gui. And the feasibility was shown to be high in Galbi gui, Galbi jjim, and Nirbiani. For the problems of the processed pork dishes feasible for export, the result showed that texture change could be problematic in Galbi gui, Samgyeopasal gui, and Nirbiani; preservation (storability) could be problematic in Bossam and Pyeonyuk; safety (food additives) could be problematic in Bulgogi and Geyook bokeum; changes in taste and flavor could be problematic in Galbi jjim and Sundae; and packaging could be problematic in Gookbob.

The results of this study, a domestic consumer prefer and think feasible for export Samgyeopasal gui, Galbi gui, Galbi jjim. Also need to future research about hygiene and safety regard to processed pork products. If improve the problems of each pork dishes, it seem to be feasible for export of Korea's traditional pork dishes.

\section{References}

1. Back, B. Y. (2000) Preferences and consumption patterns of domestic consumer to pork products. Monthly publication Young-Don, 22, 76-80.

2. Choi, I. S. (2009) Study on consumer preferences and recognition of the health benefits of pork. Master's degree thesis, Sookmyung Women's Univ., Seoul, Korea, pp. 1-48.

3. Han, J. S., Han, G. P. and Kim, T. S. (1999) A survey of recognition and use for native pork. J. East Asian Soc. Dietary Life. 9, 489-500.

4. Jeon, S. K., Park, J. Y., and Kim, Y. J. (2005) Study on the consumers' awareness on safety of livestock products and livestock processed foods. Korean J. Pract. Arts Educ. 18, 115-128.
5. Kim, E. M., Seo, S. H., Lee, M. A., Kwon, K. H., and Jun, K. H. (2010) Preferences and consumption patterns of general consumers of meat dishes. Korean J. Food Cult. 25, 251261.

6. Kim, G. W. and Kim, S. E. (2009) Analysis of the domestic consumer's preference and consumption behaviors on pork. Korean J. Anim. Sci. Technol. 51, 1-90.

7. Kim, I. S., Jang, M. B., and Back, I. G. (2000) The strategy of export extension for the pork and a processing pork product. Korean J. Food and Resources Institute 12, 48-55.

8. Kim, Y. G., Kim, J. H., Lyu, Y. M., Ahn, J. N. (2007) The common sense of meat. Nat. Ins. Anim. Sci. pp. 259.

9. Kwak, Y. T. and Ko, B. N. (2006) Pork consumption pattern analysis for non-preferred parts. Korean J. Agric. Management Policy 33, 444-455.

10. Kwak, Y. T. and Park, B. Y. (2007) The present situation of pork processing industry and plans for strengthening of competitive power. Korea J. Agric. Management Policy 34, 127147.

11. Lim, J. H. (2009) Research for increasing export of pork product. Master's degree thesis, Korea Univ., Seoul, Korea, pp. 1-68.

12. Rho, J. O., Chong, Y. K., Jung, S. J., and Cha, Y. S. (2007) A study on purchase patterns and recognition of processed foods in elementary, middle and high school meal service dietitians. Korean J. Human Ecol. 10, 63-75.

13. Seo, D. C. (2008) Analysis of the consumer's acceptability and attitudes on pork. Master's degree thesis, Kong-ju National Univ. Korea, pp. 1-29.

14. Yoon, D. R. (2003) An analysis on processed meat food marketing and consumption pattern. Master's degree thesis, Konkuk Univ., Seoul, Korea, pp. 4-56.

(Received 2011.5.27/Revised 1st 2011.12.23, 2nd 2011.12.27/ Accepted 2011.12.29) 